\title{
smarter medicine: \\ die «Top-5-Liste» der SFGG
}

Trägerschaft "smarter medicine»

\section{Choosing wisely und die Geriatrisierung der Medizin}

Die amerikanische Gesellschaft für Geriatrie hat vor einiger Zeit zehn Empfehlungen veröffentlicht im Rahmen ihrer Choosing-wisely-Initiative. Die Schweizerische Fachgesellschaft für Geriatrie (SFGG) begrüsst die geriatriespezifischen Choosing-wisely-Empfehlungen der amerikanischen Geriatrie-Gesellschaft. Es ist wichtig, dass diese auch in der Schweiz beachtet werden, in allen Institutionen, die Verantwortung tragen für die Betreuung hochaltriger Menschen.

Wir möchten Ihnen die ersten fünf Empfehlungen vorstellen. Es geht letztlich darum, grundlegende Erkenntnisse in geriatrischer Medizin auch in jenen Institutionen umzusetzen, welche (noch) nicht direkten Zugang zu Geriatrischen Fachpersonen haben.

1. Empfehlen Sie keine perkutane Magensonde bei Patienten mit fortgeschrittener Demenz; bieten Sie stattdessen eine assistierte orale Ernährung an.

Wenn Patienten mit schwerer Demenz vorsichtig von einer Pflegeperson bei der Nahrungseingabe unterstützt werden, ist dies im Hinblick auf das Sterblichkeitsrisiko, das Risiko einer Aspirationspneumonie, den funktionellen Status und den Patientenkomfort mindestens ebenso gut wie eine Sondenernährung. Die Nährstoffzufuhr erfolgt bevorzugt über normale Nahrung. Sondenernährung geht mit Unruhezuständen, dem vermehrten Einsatz von Fixierungen und medikamentöser Ruhigstellung sowie sich verschlechternden Druckgeschwüren einher.

2. Verwenden Sie Antipsychotika nicht als Mittel der ersten Wahl bei der Behandlung von Verhaltensauffälligkeiten und psychischen Symptomen bei Demenz.

Demente Patienten zeigen oft ein aggressives Verhalten, leisten gegenüber dem Pflegepersonal Widerstand oder legen andere schwierige oder störende Verhaltensweisen an den Tag. In solchen Fällen werden häufig antipsychotische Substanzen verschrieben. Diese haben jedoch nur eine begrenzte und keine verläss- liche Wirksamkeit. Gleichzeitig bergen sie das Risiko einer Übersedierung, eines kognitiven Abbaus und führen $\mathrm{zu}$ einer erhöhten Wahrscheinlichkeit von Stürzen, Schlaganfällen sowie zu einer erhöhten Mortalität. Die medikamentöse Behandlung von Demenzpatienten sollte sich auf Fälle beschränken, in denen nicht medikamentöse Therapien keine Wirkung zeigen oder die Patienten eine unmittelbare Gefahr für sich selbst oder andere darstellen. Werden die Ursachen für die Verhaltensänderungen ermittelt und angegangen, kann dies die medikamentöse Behandlung ersetzen.

\section{Breite Unterstützung und neuer Schub \\ für die Kampagne smarter medicine}

Ein neu gegründeter Trägerverein verleiht der 2014 in der Schweiz lancierten Initiative smarter medicine Aufwind: Nebst medizinischen Fach- und Berufsorganisationen unterstützen auch Patienten- und Konsumentenorganisationen die Stossrichtung der Kampagne. Sie möchten gemeinsam die Öffentlichkeit dafür sensibilisieren, dass bei gewissen Behandlungen weniger Medizin mehr Lebensqualität für die Betroffenen bedeuten kann.

Sie knüpfen dabei an die erfolgreiche amerikanische Initiative "Choosing Wisely" an, welche zum Ziel hat, nicht nur «kluge Entscheidungen» herbeizuführen, sondern auch die offene Diskussion zwischen Ärzteschaft, den Patienten und der Öffentlichkeit zu fördern. In den nächsten Monaten werden zudem verschiedene medizinische Fachgesellschaften sogenannte Top-5-Listen mit unnützen Behandlungen in ihrem Fachbereich publizieren. Den Auftakt machen die Empfehlungen in der Intensivmedizin und in der Geriatrie, welche die zwei bestehenden Listen in der Inneren Medizin (ambulant und stationär) ergänzen.

Weitere Informationen zum Trägerverein und eine Übersicht über die bestehenden Top-5-Listen findet man unter www.smartermedicine.ch 
3. Vermeiden Sie bei den meisten älteren Erwachsenen die Gabe anderer Medikamente als Metformin, um einen Hämoglobin-A1c-Wert (HbA1c) von unter 7,5\% zu erreichen; eine moderate Blutzuckerkontrolle ist im Allgemeinen besser.

Es gibt keine Hinweise, dass der Einsatz von Medikamenten zum Erreichen einer straffen Blutzuckerkontrolle bei den meisten älteren Patienten mit Typ2-Diabetes einen positiven Effekt hat. Bei weniger älteren Patienten wird die medikamentöse Behandlung zur Erreichung eines glykosilierten Hämoglobinspiegels von unter 7,0\% mit Schädigungen, einschliesslich höherer Mortalitätsraten, in Verbindung gebracht. Eine Ausnahme bildet die langfristige Senkung der Gefahr eines Herzinfarkts und der Sterblichkeit durch die Gabe von Metformin. Es hat sich immer wieder gezeigt, dass eine strenge Kontrolle des HbAlc-Spiegels bei älteren Menschen vermehrt zu einer Unterzuckerung führt. Bedenkt man zudem die lange Zeitspanne, die notwendig ist, um mit einer strikten Blutzuckerkontrolle mögliche theoretische mikrovaskuläre Vorteile zu erzielen, sollten bei der Blutzuckereinstellung die Patientenziele, der Gesundheitszustand sowie die Lebenserwartung berücksichtigt werden. Empfohlen werden folgende HbA1c-Werte: 7,0 bis 7,5\% bei gesunden älteren Patienten mit langer Lebenserwartung; 7,5 bis 8,0\% bei Patienten mit moderater Anzahl Begleiterkrankungen und einer Lebenserwartung von $<10$ Jahren; 8,0 bis $9,0 \%$ bei Patienten mit Mehrfacherkrankungen und kürzerer Lebenserwartung.

4. Verwenden Sie bei älteren Erwachsenen keine Benzodiazepine oder andere sedativ-hypnotischen Arzneien als Mittel der ersten Wahl gegen Schlaflosigkeit, Unruhezustände oder Verwirrtheit.

Grosse Studien zeigen immer wieder, dass sich das Risiko für Verkehrsunfälle, Stürze und Hüftfrakturen sowie für Hospitalisierungen oder Tod bei älteren Menschen mehr als verdoppeln kann, wenn Benzodiazepine oder andere Beruhigungs- oder Schlafmittel verordnet werden. Ältere Patienten und das Gesundheitspersonal sollten die potentiellen Gefahren der verschiedenen Behandlungsstrategien für Schlaflosigkeit, Unruhezustände oder Verwirrtheit kennen. Die Anwendung von Benzodiazepinen sollte auf Alkohol-

\section{Zur Ausarbeitung dieser Liste}

Die American Geriatrics Society (AGS) hat eine Arbeitsgruppe unter der Leitung des stellvertretenden Vorsitzenden des Clinical Practice and Models of Care Committee (CPMC) ins Leben gerufen. Die Arbeitsgruppe setzte sich aus Mitgliedern dieses Ausschusses sowie des Ethik- und Ethnogeriatrieausschusses und des Quality and Performance Measurement Committee (OPMC) zusammen. Mitglieder der AGS wurden via elektronische Umfrage gebeten, Feedback und Empfehlungen darüber abzugeben, welche Punkte in die Liste aufgenommen werden sollten. In einem ersten Schritt kürzte die Arbeitsgruppe die Liste auf die zehn wichtigsten potenziellen Tests oder Verfahren. Dann überprüfte sie die jeweiligen Erkenntnisse, zog Sachverständige zu Rate, um die Liste weiter auf fünf Empfehlungen zu verdichten. Diese wurden schliesslich vom AGS Executive Committee und den Vorsitzenden/stellvertretenden Vorsitzenden des CPMC, des Ethikausschusses und des QPMC geprüft und genehmigt.

Der Vorstand der Schweizerischen Fachgesellschaft für Geriatrie (SFGG) hat die Empfehlungen der amerikanischen Gesellschaft für Geriatrie im Rahmen einer Vorstandssitzung geprüft und ist dabei zum Schluss gekommen, dass diese auch für die Schweizer Verhältnisse zutreffend und relevant sind.

Eine ausführliche Literaturliste sowie Empfehlungen von weiteren Medizinischen Fachgesellschaften sind unter www.smartermedicine.ch online abrufbar.

entzugserscheinungen / Delirium tremens oder schwere generalisierte Angststörungen, die nicht auf andere Therapien ansprechen, beschränkt sein.

5. Verwenden Sie keine Antibiotika gegen Bakteriurie bei älteren Erwachsenen, ausser es liegen spezifische Harnwegssymptome vor.

Kohortenstudien haben keine negativen Auswirkungen einer asymptomatischen Bakteriurie bei älteren Frauen und Männern nachweisen können. Und während die antimikrobielle Behandlung selbst keine Vorteile mit sich bringt, nehmen die unerwünschten $\mathrm{Ne}$ benwirkungen einer solchen Behandlung bei älteren Menschen zu. Es wurden Konsenskriterien erarbeitet, anhand derer sich die spezifischen Symptome erkennen lassen, die in Verbindung mit einer Bakteriurie für einen Harnwegsinfekt sprechen. Im Vorfeld urologischer Eingriffe, bei denen mit Schleimhautblutungen gerechnet wird, empfiehlt sich eine Untersuchung auf asymptomatische Bakteriurie und deren Behandlung. 